\title{
Rating Burn Wounds by Dynamic Thermography
}

\author{
by M. Kaczmarek*, A. Nowakowski, A. Renkielska** \\ *Department of Medical and Ecological Electronics, Technical University of Gdansk, \\ Narutowicza 11/12, 80-952 Gdansk, e-mail:mariusz@biomed.eti.pg.gda.pl, antowak@pg.gda.pl \\ ** Department of Plastic Surgery and Burns, Medical Academy of Gdansk, \\ M. Curie-Sklodowskiej 3A, 80-210 Gdansk, Poland
}

\begin{abstract}
Use of the dynamic thermography (lock-in and pulse) for assessment of burns is discussed. Measurements of burns under medical treatment with different depth of affected tissue (from the first to the third-degree burns) are shown. The thermographic data are correlated with histopathological analysis of lesions. The results show that dynamic thermography might be advice as a simple, noninvasive and non-stressed for patients diagnostic tool. Further analysis of dynamic pictures gives the first estimate of the depth of a lesion.
\end{abstract}

\section{Introduction}

A reliable, objective, universal and non-invasive method for assessing burns or postoperative scars does not exist in today's clinical practice. Such a method would be useful for making proper diagnosis and taking decisions about further treatment. Some attempts on decision making in the treatment of burns were made, for example: Static Thermography [1], Laser Doppler Imaging [2], Reflection-Optical Multispectral Imaging Method [3], but none of these techniques is widely accepted in clinical practice.

The method should be based on measurable, objective tissue properties - this might be performed studying such figures of merit as thermal conductivity or thermal capacitance. Values of these parameters are related to physiological state of skin and subdermal tissues, its vascularity, pliability and maturation. Moreover, it is necessary to see progress of a treatment, especially in a case of complications, for prompt reaction and necessary changes of therapy procedures. We studied if dynamic thermography might be used as a useful tool for such investigations.

\section{Theory}

Burns typically are caused by one of many types of interactions of tissue with high temperature sources (flames, hot solids and liquids); with electrical energy dissipation; with high energy ionising radiation (X-ray, nuclear, UV radiation); with chemical substances (acids, bases, gas warfare agents) and other. All of these burns have specific character of injury followed by a healing process. The stage (depth) of an injury depends on a specific phenomenon and may be developed in time (especially concerns chemical or irradiation lesions).

In clinical practice the burns are divided into:

- the first-degree burns - the major change is vasodilatation of sub-papillary vessels in the affected area and the reddening of the tissue; the healing process is connected with migration of cells to the epithelial surface. No scars and discoloration is observed after healing process;

- the second-degree burns (partial thickness lesion) - evidenced in capillary damage resulting in blisters formation. The cells may become swollen or even damaged. There is a very high concentration of water and albumin in the extra-cellular spaces. Superficial (IIA) and deep second-degree (IIB) burns can be identified. Superficial injury is healing spontaneously without scaring (basal cells of the corium are not destroyed) but with possible depigmentation. In deep burns - IIB - a lot of the basal corium cells is lost; the 
vascular system is occluded and stagnation in the blood flow in the tissue is observed. Blistering is not widespread. Healing is difficult - a wound becomes resurfaced from undamaged epithelial cells in the hair follicles and from the margins of the injured area. The new epithelium is thinner comparing to normal one and the skin is lighter due to permanent loss of the melanocytes;

- the third-degree burns (full-thickness lesion) - all epidermal elements and supporting dermal structures are destroyed. The blood flow is stopped in the microcirculation system. The local nervous system is destroyed. No possibility for spontaneous healing of the lesion exists. Resurfacing occurs only from the margin of the healthy skin or by application of a skin graft;

- the fourth-degree burns - it is evident as an incineration of tissue from skin to muscle and even bones.

It is very important to know if a surgery and skin-grafting procedure is necessary or a wound will be healing spontaneously. So, the question is: what we see - a IIB or a III degree burn or a less danger case? This information is necessary as early as it is possible. A delay in treatment can cause complications and bacterial infections. The cardinal problem in determining of a burn degree is the variability of a skin type (e.g. old one or young one) and different effects of injury due to a source of burn (flame, chemicals, liquids, etc.). There are also different properties of skin in different body areas - skin thickness can vary from millimetres on hands, up to centimetres on the back. External view of an injured area is not effective for determination of a depth of a lesion.

Active thermography using external irradiation may be used for determination of a depth of a burn. The initial assumptions applied for dynamic thermography procedures in skin examination are:

- the skin is a 2- or 3-dimensional medium with at least 3-layer composite structure (epidermis, dermis, subcutaneous tissue) - see fig.1;

- $\quad$ tissue properties are independent on it's initial temperature;

- external excitation is non-invasive.

Temperature distribution and it change in time during and after excitation can be described by bio-heat transfer equation [4]:

$$
c_{t} \rho_{t} \frac{\partial T(x, y, z, t)}{\partial t}=k \nabla^{2} T(x, y, z, t)+Q_{b}+Q_{m}+Q_{z},
$$

where: $c_{t}$ - specific heat, $\rho_{t}$ - mass density, $\nabla^{2} T(x, y, z, t)$ - volumetric power density exchanged due to temperature gradient and thermal conduction, $\mathrm{Q}_{b}$ - volumetric power density exchanged due to blood perfusion, $\mathrm{Q}_{m}$ - volumetric power density caused by metabolism, $\mathrm{Q}_{z}$ - volumetric dissipated power density of an external heating source (e.g. optical, microwave or RF).

It is easy to see that for the II degree burns the components $Q_{b}$ and $Q_{m}$ are reduced, and $\nabla^{2} T(x, y, z, t)$ is modified according to high concentration of water and albumin in the extracellular spaces, whereas for the III degree burns the components $Q_{b}$ and $Q_{m}$ are absent. So, for a uniform power density field formed by an external source $Q_{z}$ a temperature distribution on a body surface will be correlated with a physical state of skin tissues.

\section{Method}

The related burn injuries were formed in different accidents as a result of flame action directly on skin - hands and faces - and on burning clothes - thorax. Only two cases of burns are related although we have examined many more (around 100) patients with different skin injuries. The investigations were performed during the first 5 days following an accident. Long lasting effects of treatment and scars formation are still studied and not related here, yet. 
For dynamic thermography experiments (lock-in and pulse) the Agema 900 system with specially developed software and excitation hardware using halogen lamps - 500 or $1000 \mathrm{~W}$ of electrical power - remotely illuminating the observation area comprising an injured and healthy tissue are applied. Investigations were performed for different frequency modulation $-0.1 ; 0.05 ; 0.025 \mathrm{~Hz}$ - for the lock-in procedure and for different pulse duration - from 10 up to 30 seconds - for the pulse thermography. Illuminated area was kept wet using a thin layer of ointment to satisfy condition of constant evaporation from a tested surface and a constant value of emissivity coefficient.

For comparison photographs, thermograms and dynamic parametric images - for lock-in thermography - the phase and the magnitude images, for pulsed thermography - NDPTI (Normalised Difference Pulsed Thermography Index [5]) images are analysed.

\section{Discussion}

The comparison of a reaction to external irradiation of healthy and affected tissue is of major importance. It is also very important to set a proper power density and an exposure time because burn injuries are very sensitive to increase of temperature - it is important to avoid any pain during the test. Generated thermal wave is absorbed and transmitted by tissues accordingly to physiological conditions and physical (mechanical) structure. Unfortunately for further image processing, the human body is a very difficult object for inspection. Breathing, spontaneous movements and other artefacts can disturb the evaluated results. Also, heat exchange processes at the outer surface is complicated due to conduction, convection, radiation and evaporation resulting in a very complex model of heat distribution. Therefore here only burns of thorax which gives the most reliable results for evaluation of the proposed method are discussed. Regular shape of a tested area and uniform skin thickness of flat parts of the body allows determination of the relationship between a state of the skin (burn degree) and thermographic data. Possible artefacts and interactions with bones, large arteries and veins lying under a tested surface are excluded. In the next step when the method will be well matched we hope it will be possible to eliminate such artefacts existing in other parts of the body by image processing.

The moment of thermographic investigation after an accident is of the highest significance. To our experience the most valuable results are obtained during the first and the second day following an accident. On figures $2 \mathrm{a}, \mathrm{b}, \mathrm{c}$ classical passive thermograms of burn wounds of the patient 1 at the second day and the fifth day following the accident are shown - $2 b$ - before removing the dead skin; - $2 c$ - after removing the old (dead) skin. The dead layer of skin disturbs reading of the true temperature distribution on the skin surface. The isotherm $36.1^{\circ} \mathrm{C}$ is denoted by the green colour. As one can see the fields of the same temperature are quite different in both cases. This is worth to mention that blisters cause the same effect but as it was discussed earlier the IIB and III degree burns are without blisters.

The figures 3 - 6 show photographs and thermographic data results of the same patient done by lock-in and pulsed thermography. Interpretation of the data is difficult but possible when additional measurements of the properties of the wounded tissue are available. Generally more informative for burn quality evaluation is the phasogram. In the magnitudogram some picture features are strongly dependent on geometry (curvatures) of a tested area not related to burn quality. Photograph 5 shows that the healing process was very effective and the affected field is recovering in a fast way. No skin grafts were necessary.

Figures $7-10$ show photographs and thermographic data of the second patient - burns caused by fire. Well defined fields of III degree burns are shown on both - pulse and lock-in thermograms. The effect of treatment is also very well visible comparing the results of measurements done day by day. The burn area was enough small to avoid grafting as in the case of the first patient. The lower frequency $(0.01 \mathrm{~Hz})$ pictures are more informative, too.

For both studied cases the histopathological examination was done. For the first case the second-degree burn was recognised and confirmed during medical treatment. In the second case a piece of skin cut for histopathological examination showed how thick was the burn 
(the cut of tissue for histopathological examination was done at the centre of the most affected region of the burn). Thermographic parametric images (NDPTI and phase) indicated regions of evidently changed features very well correlated to the III degree burn.

\section{Conclusions}

The main goal of the research - to find if the active thermography may be applicable for burn diagnostics - was verified. The following step is to find not only qualitative information but also quantitative one, to describe ratings of the burn wounds.

Changes in dermal tissue vascularisation are important for prediction of treatment progress. We proved that the dynamic thermography can by applied in medical procedures for monitoring the state of the skin and subdermal tissue structure during burns treatment giving objective, measurable ratings of the treatment procedure. In our opinion the clinical valuable features of the method are:

- the early objective determination of the burn depth - up to two days after an accident,

- the non-contact, non-invasive, clean and non-stressed method,

- one shoot wide area investigation,

- $\quad$ the support for decision if it is the II-nd or the III-rd degree injury,

- clear and objective documentation of diagnoses and treatment process.

The work is still under development. The next step will be an in vivo experiment on animals (pigs whose skin has very similar properties as human skin) with fully controlled conditions of injury. Also special software is under development for objective and automatic generation of injury depth maps. The other problem to be solved is development of a method for non-contact determination of basic properties of thermal parameters of affected tissue.

Acknowledgements - this work is financed by KBN grant Nr 8T11E03015

\section{References}

[1] Liddington M. I., Shakespeare P. G., Timing of the thermographic assessment of burns, Burns Vol. 22, No 1, 1996,

[2] Brown R. F. R., Rice P., Bennett N. J., The use of laser Doppler imaging as an aid in clinical management decision making in the treatment of vesicant burns, Burns Vol. 24, No 6, 1998,

[3] Eisenbeis W., Marotz J., Schrade J. P., Reflection-optical multispectral imaging method for objective determination of burn depth, Burns Vol. 25, No 6, 1999,

[4] Pennes H. H., Analysis of tissue and arterial blood temperatures in the resting human forearm, J. Appl. Physiol, No 93, 1948,

[5] Kaczmarek M., Nowakowski A., Measurement of Thermal Properties of Biological Tissues - Comparison of Different Thermal NDT techniques, $5^{\text {th }}$ International Workshop on Advanced Infrared Technology and Applications, Venice, 1999,

[6] Shitzer A., Eberhart R. C., Heat transfer in medicine and biology, Plenum Press, New York, London, 1985.

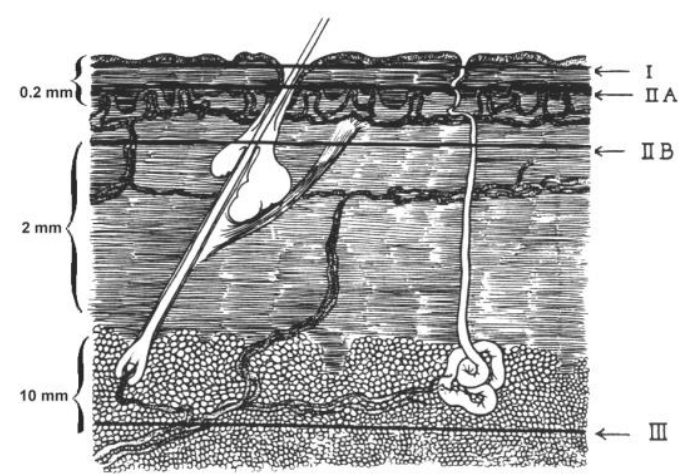

Figure 1. Structure of skin - depths of destroyed cells according to burn degree are shown [6] 


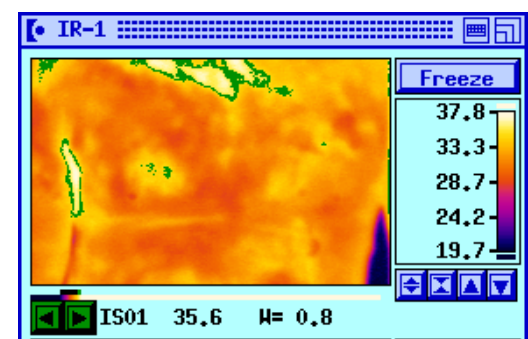

a) the second day after the accident

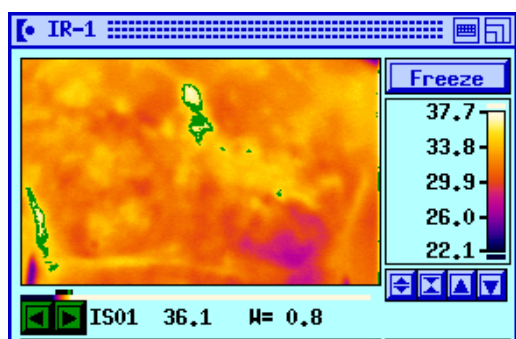

b) the fifth day after the accident

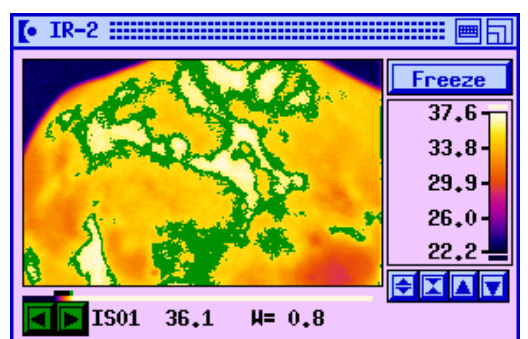

c) as b) but after the dead skin is and before dead skin is taken off taken off

Figure 2. Thermograms of the patient 1 - a burned chest; the same isotherm is indicated by green on b) and c)

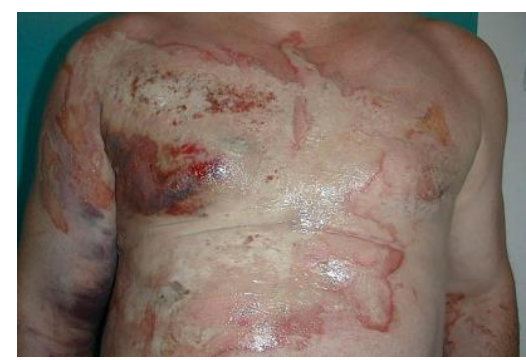

a)

Figure 3. Photo of the patient 1 - the second day after the accident (a), pulse thermography - NDPTI images for time 60s (b) and $120 \mathrm{~s}$ (c)

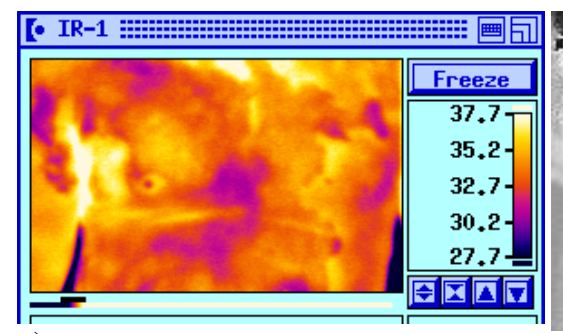

a)

\section{b)}
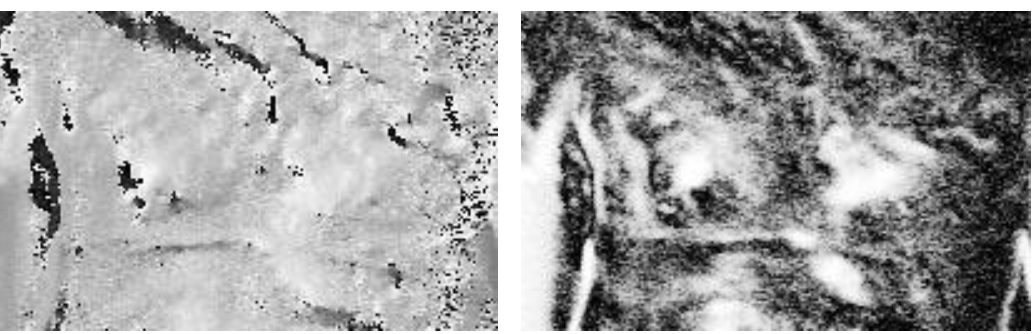

c)

Figure 4. Patient 1 - second day after the accident (as on fig.3) - the thermogram (a); lock-in thermography for frequency modulation $\mathrm{f}=0.025 \mathrm{~Hz}$ - the phase image (b) and the magnitude image (c)
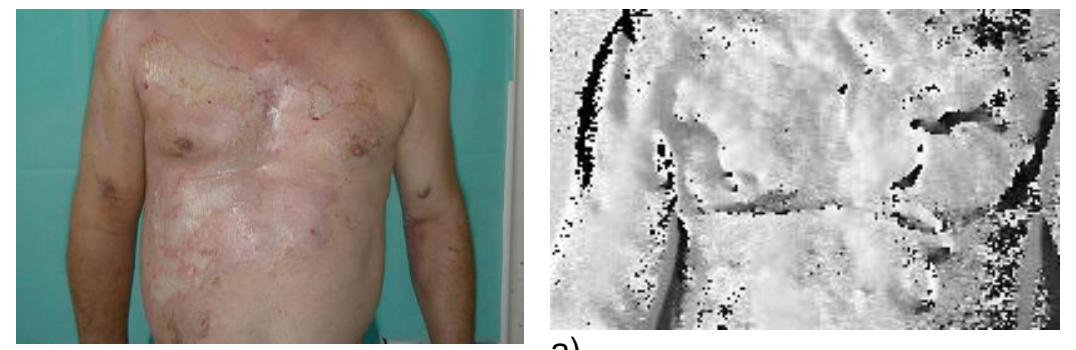

a)

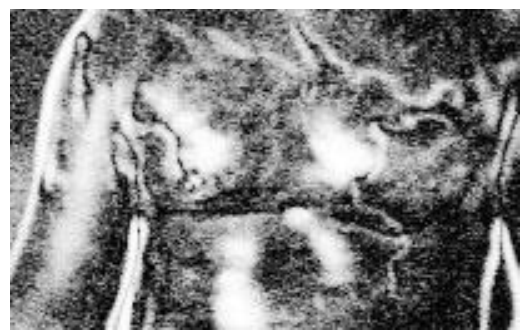

b)

Figure 5. Picture of the patient 1 Figure 6. Patient 1 - lock-in investigation at the third day after the taken ten days after the accident accident for frequency modulation $\mathrm{f}=0.025 \mathrm{~Hz}$; the phase image (a) and the magnitude image (b) 


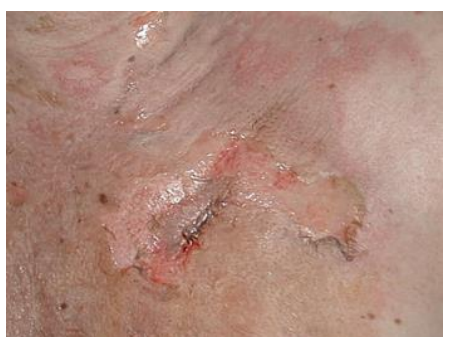

a)

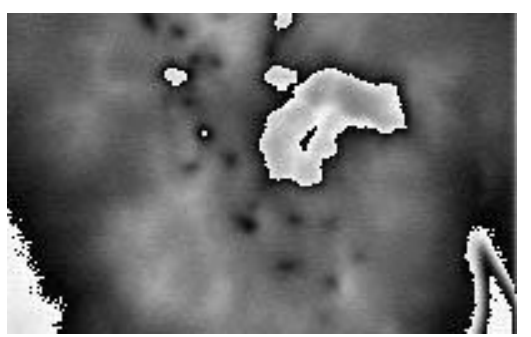

b)

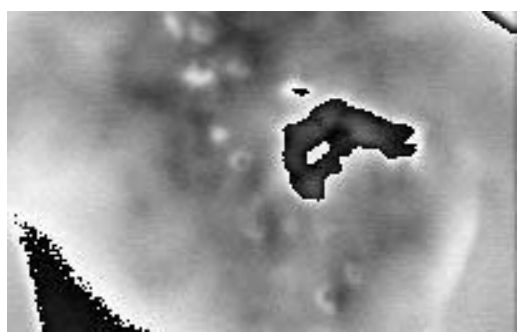

c)

Figure 7. Patient 2, the first day after the accident - photograph of the burn (a); NDPTI image of the same field (b); the NDPTI image at the second day (c)

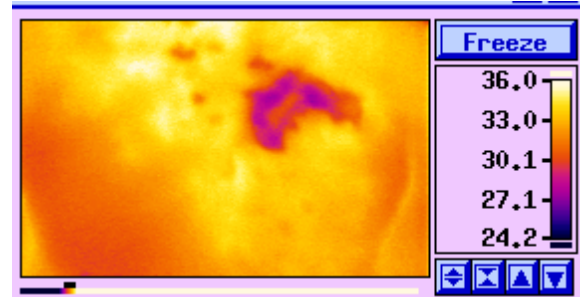

a)

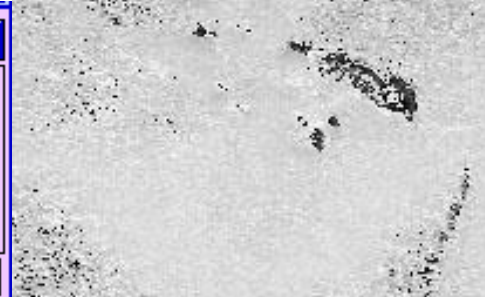

b)

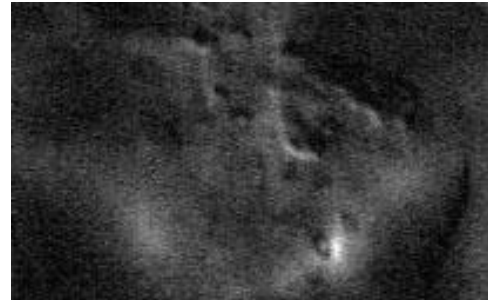

c)

Figure 8. The same case as on fig. 7, the first day after the accident - thermogram (a); lock-in data for frequency modulation $\mathrm{f}=0.05 \mathrm{~Hz}$ (b) - the phase image; (c) - the magnitude image

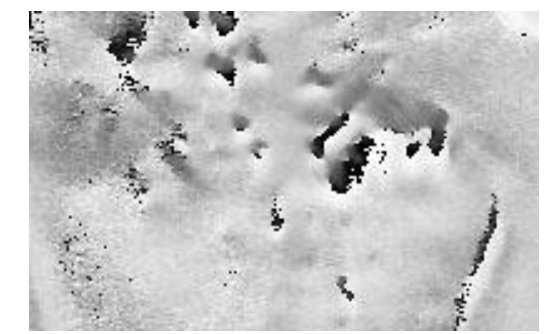

a)

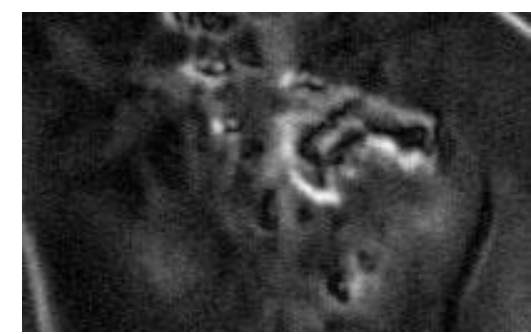

b)

Figure 9. The same case as on fig. 7 , the first day after the accident - lock-in data for $\mathrm{f}=0.01 \mathrm{~Hz}$ - the phase image (a); the magnitude image (b) - the injured field is better defined comparing to the fig. 8

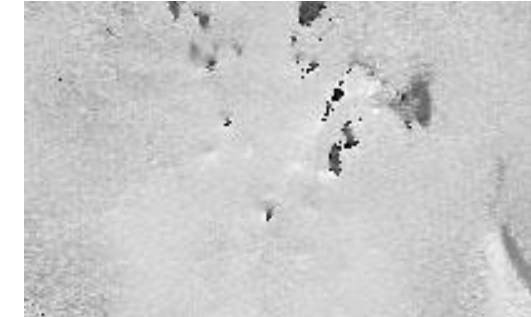

a)

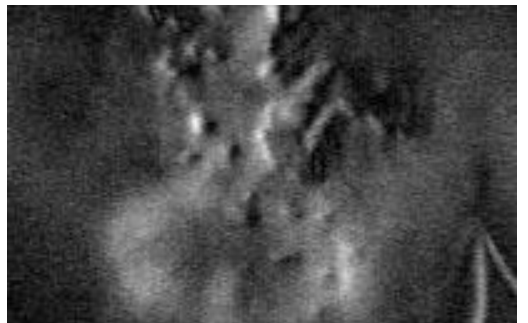

b)

Figure 10. As fig. 9, the second day after the accident - lock-in data for $f=0.01 \mathrm{~Hz}$ - the phase image (a) and the magnitude image (b) - the healing process is already evident 